13

\title{
Совмещение конфигураций Отто и Кретчмана для возбуждения различных мод поверхностных плазмон-поляритонов
}

\author{
() С.В. Томилин, А.В. Каравайников, О.А. Томилина, В.Н. Бержанский
}

Физико-технический институт, Крымский федеральный университет им. В.И. Вернадского, 295007 Симферополь, Россия

e-mail: tomilin_znu@mail.ru

Поступило в Редакцию 14 апреля 2020 г.

В окончательной редакции 4 сентября 2020 г.

Принято к публикации 7 сентября 2020 г.

Представлены результаты моделирования, синтеза и исследования свойств двухрезонансной плазмонной структуры для возбуждения различных мод поверхностных плазмон-поляритонов. Показана принципиальная возможность возбуждения плазмон-поляритонных резонансов на основе совмещения конфигураций Кретчмана и Отто на одной асимметричной многослойной структуре. Выполнена оптимизация параметров такой структуры для получения максимальной эффективности возбуждения поверхностных плазмон-поляритонов. Рассмотрены возможности применения предложенной двухрезонансной плазмонной структуры в качестве самостоятельного плазмон-поляритонного сенсора и в роли чувствительного элемента плазмонного биосенсора.

Ключевые слова: вакуумное напыление, тонкая пленка, плазмон-поляритонный резонанс, конфигурация Кретчмана, конфигурация Отто, двухрезонансная плазмонная структура.

DOI: 10.21883/JTF.2021.02.50371.132-20

\section{Введение}

Принцип действия датчиков на поверхностных плазмон-поляритонах (ППП) основан на физическом эффекте изменения резонансной частоты поверхностных плазмон-поляритонных волн в зависимости от диэлектрической проницаемости среды, граничащей с чувствительным металлическим (плазмонным) слоем [1-6]. Существует две классические конфигурации призменного возбуждения поверхностных плазмон-поляритонных волн $[1,7,8]$. Первая, конфигурация Кретчмана, характерна тем, что плазмонный слой металла (как правило, используются $\mathrm{Cu}, \mathrm{Ag}, \mathrm{Au})$ наносится непосредственно на гипотенузную грань призмы полного внутреннего отражения. При этом слой плазмонного металла выполняется очень тонким (порядка 30-60 nm), а плазмонполяритонная волна возбуждается на границе раздела металлического покрытия и окружающего пространства $[8,9]$. Вторая, конфигурация Отто, отличается от предыдущей тем, что плазмонный слой металла не наносится на поверхность призмы, а прижимается к нему, при этом между призмой и металлом остается тонкий слой диэлектрика с показателем преломления меньше, чем у призмы [7]. В данной конфигурации особое внимание уделяется толщине диэлектрического слоя (порядка $100-250 \mathrm{~nm}$ ), при этом плазмонный слой может быть принципиально любой толщины более $20 \mathrm{~nm}$.

Авторами настоящей работы разработана плазмонная двухрезонансная структура на основе призмы полного внутреннего отражения, позволяющая совместить конфигурации Кретчмана и Отто для возбуждения двух различных мод ППП (рис. 1) [10]. Особенность та- кой плазмонной структуры состоит в том, что между призмой и плазмонной металлической пленкой располагается дополнительный (буферный) слой прозрачного диэлектрика с показателем преломления меньшим, чем у призмы, но большим, чем у окружающей среды. Таким образом, полное внутреннее отражение (ПВО) на границе диэлектрического слоя и окружающей среды возникает при малых углах падения луча, при этом возбуждается плазмон-поляритонная волна на внешней поверхности плазмонной пленки аналогично конфигурации Кретчмана (резонанс по Кретчману). При больших углах падения луча ПВО наблюдается на границе призмы и диэлектрического слоя, при этом возбуждается плазмон-поляритонная волна на внутренней поверхности плазмонной пленки аналогично конфигурации Отто (резонанс по Отто).

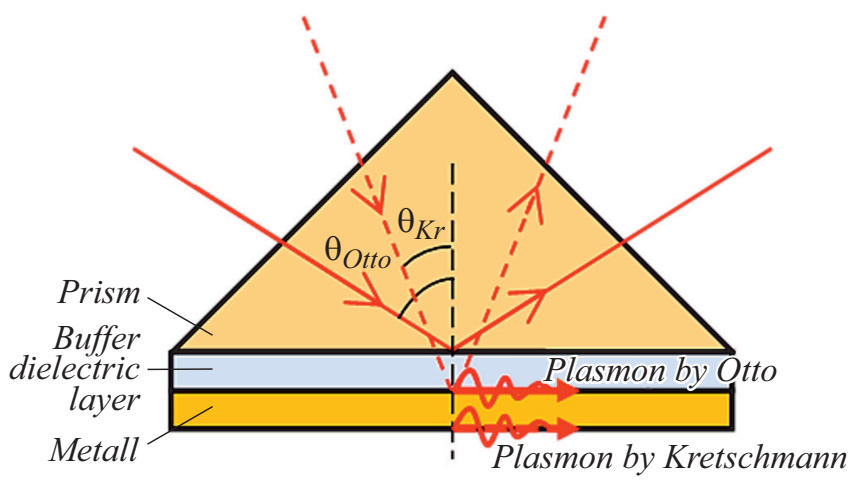

Рис. 1. Схема предложенной двухрезонансной плазмонной структуры для возбуждения различных мод ППП. 
Для предложенной структуры на угловой рефлектометрической зависимости будут наблюдаться два интенсивностных провала (пика), связанных с резонансным возбуждением различных мод ППП (по Кретчману и по Отто соответственно), при этом условие резонанса по Кретчману будет существенно зависеть от диэлектрических свойств окружающей среды, что позволяет реализовать сенсорную систему для детектирования изменения свойств этой среды. Условие резонанса по Отто (и его положение на рефлектометрической кривой), напротив, зависит в основном от свойств диэлектрического буферного слоя и соответственно изменяться будет слабо. Такой резонанс можно использовать как опорный при сравнении с изменением положения резонанса по Кретчману, что позволит повысить точность и стабильность работы двухрезонансной структуры в режиме сенсора по сравнению с однорезонансной. Отметим, что другими исследовательскими группами также предпринимаются подобные шаги по созданию двухрезонансных плазмонных систем, совмещающих резонансы Отто и Кретчмана, например, на спектральных датчиках оптоволоконного типа с буферным слоем, имеющим сильную дисперсию диэлектрической проницаемости [11].

Таким образом, целью настоящей работы является теоретический (модельный) и экспериментальный анализ оптимальных параметров и условий возбуждения различных мод поверхностных плазмон-поляритонов при совмещении конфигураций Отто и Кретчмана в одной асимметричной многослойной структуре, а также исследование возможностей использования такой структуры в качестве чувствительного элемента плазмонного сенсора.

\section{1. Методы получения и исследования образцов}

Разработанную двухрезонансную структуру для возбуждения различных мод ППП предлагается реализовать на основе оптической схемы, в которой используется прямоугольная призма полного внутреннего отражения из монокристалла гадолиний-галлиевого граната $\mathrm{Gd}_{3} \mathrm{Ga}_{5} \mathrm{O}_{12}\left(\mathrm{GGG}_{\text {prism }}\right)$ с высоким показателем преломления $n=1.960$ для $\lambda=632.8 \mathrm{~nm}$, на которую напыляется буферный слой диэлектрика толщиной 20-350 nm (с градиентом толщины) с показателем преломления меньшим, чем у призмы, но большим, чем у окружающей среды (кварц $\mathrm{SiO}_{2}, n=1.456$ для $\lambda=632.8 \mathrm{~nm}$ ). Поверх буферного слоя наносится плазмонная металлическая пленка $\mathrm{Au}$ чистотой не хуже 99.95\%.

\section{1. Численное моделирование и оптимизация параметров двухрезонансной плазмонной структуры}

Численное моделирование структуры для возбуждения различных мод ППП, а также оптимизация ее струк- турных параметров и режимов работы были выполнены с использованием программного пакета WinSpall. При этом рассчитывается нормированная интенсивность отраженного луча (френелевский коэффициент отражения $R p$ для $p$-компоненты возбуждающего монохроматического пучка) в зависимости от угла падения (угловая рефлектометрия). В качестве модели была выбрана конфигурация с треугольной призмой полного внутреннего отражения (углы 90, 45, 45). Диэлектрическая проницаемость материала призмы $\varepsilon_{\mathrm{GGG}}=3.842$, что соответствует гадолиний-галлиевому гранату на длине волны $\lambda=632.8 \mathrm{~nm}$ (мнимая часть $\varepsilon_{\mathrm{GGG}}$ в данном случае опускается, поскольку в рамках модели мы пренебрегаем потерями излучения в призме). Соответствующие параметры для буферного слоя: $\varepsilon_{\mathrm{SiO}_{2}}=2.122+0.0001 i$ (кварц $\mathrm{SiO}_{2}$ ), толщина $h_{\mathrm{SiO}_{2}}$ варьируется в диапазоне от 100 до $300 \mathrm{~nm}$ для оптимизации параметров. Параметры плазмонного слоя (пленка золота): $\varepsilon_{\mathrm{Au}}=-11.647+1.263 i$, толщина $h_{\mathrm{Au}}$ также варьируется от 30 до $70 \mathrm{~nm}$. Диэлектрическая проницаемость внешней среды $\varepsilon_{\text {env }}$ варьируется в диапазоне от 1 до 2 с целью оптимизации параметров работы чувствительного элемента сенсора. Кроме того, рассматривается модель, в которой поверх плазмонного слоя располагается дополнительный слой диэлектрика, такая конфигурация моделирует условия работы двухрезонансной структуры в режиме биосенсора [12], где свойства чувствительного слоя (лиганда Lg) изменяются посредством варьирования его параметров (диэлектрическая проницаемость $\varepsilon_{\mathrm{Lg}}$ изменяется в диапазоне от 1 до 2 , а толщина $h_{\mathrm{Lg}}$ - от 0 до $300 \mathrm{~nm})$.

\section{2. Изготовление плазмонной структуры для возбуждения различных мод ППП}

Двухрезонансная структура, совмещающая в себе конфигурации Отто и Кретчмана для возбуждения различных мод ППП, была изготовлена методами вакуумного напыления. Напыление буферного слоя $\mathrm{SiO}_{2}$ на гипотенузную грань призмы из монокристаллического GGG осуществлялось методом ионно-реактивного распыления кварцевой мишени в среде аргона и кислорода. При напылении применялась методика формирования покрытия с градиентом толщины $h_{\mathrm{SiO}_{2}}$ [13]. Направление градиента $h_{\mathrm{SiO}_{2}}$ выбрано перпендикулярно к треугольной грани призмы.

Напыление плазмонного слоя Аu выполнялось методом термического испарения в вакууме исходной загрузки $\mathrm{Au} \mathrm{c} \mathrm{чистотой} \mathrm{99.95 \%} \mathrm{из} \mathrm{молибден-вольфрамового}$ тигля. Остаточное давление в рабочей камере не превышало $6 \cdot 10^{-4} \mathrm{~Pa}$.

Измерение толщины напыляемых слоев выполнялось на образце-свидетеле с помощью микроинтерферометра Линника МИИ-4 с блоком цифровой обработки. Исследование особенностей возбуждения различных мод ППП в изготовленной двухрезонансной структуре осуществлялось с использованием оптической системы на 

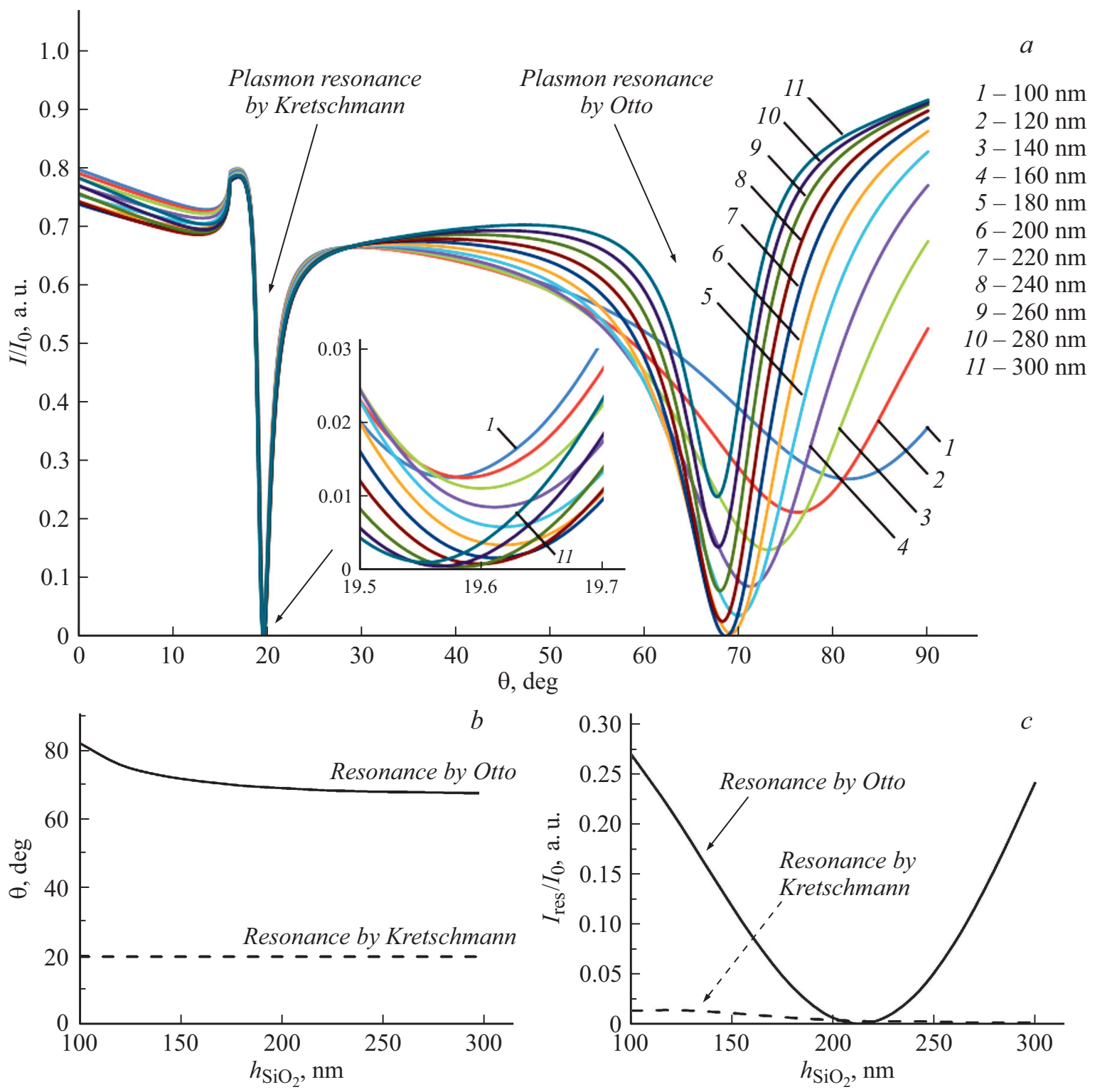

Рис. 2. Влияние толщины буферного слоя $h_{\mathrm{SiO}_{2}}$ на резонансные свойства плазмонной структуры $\mathrm{GGG}_{\mathrm{prism}} / \mathrm{SiO}_{2} / \mathrm{Au}(50): a-\mathrm{yг-}$ ловые зависимости интенсивности отраженного луча $I$, нормированной на интенсивность падающего луча $I_{0}\left(h_{\mathrm{SiO}_{2}}\right.$ указана в легенде); $b$ - зависимость резонансных углов $\theta_{\mathrm{Kr}}\left(h_{\mathrm{SiO}_{2}}\right)$ и $\theta_{\mathrm{Otto}_{0}}\left(h_{\mathrm{SiO}_{2}}\right) ; c$ - нормированные интенсивности резонансных сигналов $I_{\text {res }}\left(h_{\mathrm{SiO}_{2}}\right) / I_{0}$.

базе нуль-эллипсометра ЛЭФ-3М-1. Исследования проводились в различных участках градиента толщины буферного слоя с шагом вдоль градиента $3 \mathrm{~mm}$, что соответствует изменению $h_{\mathrm{SiO}_{2}}$ примерно на $40-60 \mathrm{~nm}$.

\section{2. Результаты и их обсуждение}

\section{1. Оптимизация параметров двухрезонансной структуры для возбуждения различных мод ППП}

Оптимизация параметров двухрезонансной структуры для возбуждения различных мод ППП осуществлялась путем моделирования влияния толщины буферного слоя $\mathrm{SiO}_{2}$ и толщины плазмонного слоя $\mathrm{Au}$ на особенности и условия возбуждения плазмонных резонансов.

Результаты влияния толщины буфера $\mathrm{SiO}_{2}$ на плазмонные свойства двухрезонансной структуры представлены на рис. 2. В частности, на рис. 2, $a$ представлены кривые зависимости интенсивности отраженного луча от угла падения (угловая рефлектометрия) при разной толщине буферного слоя. Минимумы на кривых соответствуют поглощению световой энергии при резонасном возбуждении поверхностных (распространяющихся) плазмонполяритонных волн. Как было сказано выше, при малых углах падения реализуется резонансное возбуждении ППП в соответствии с конфигурацией Кретчмана, а при 

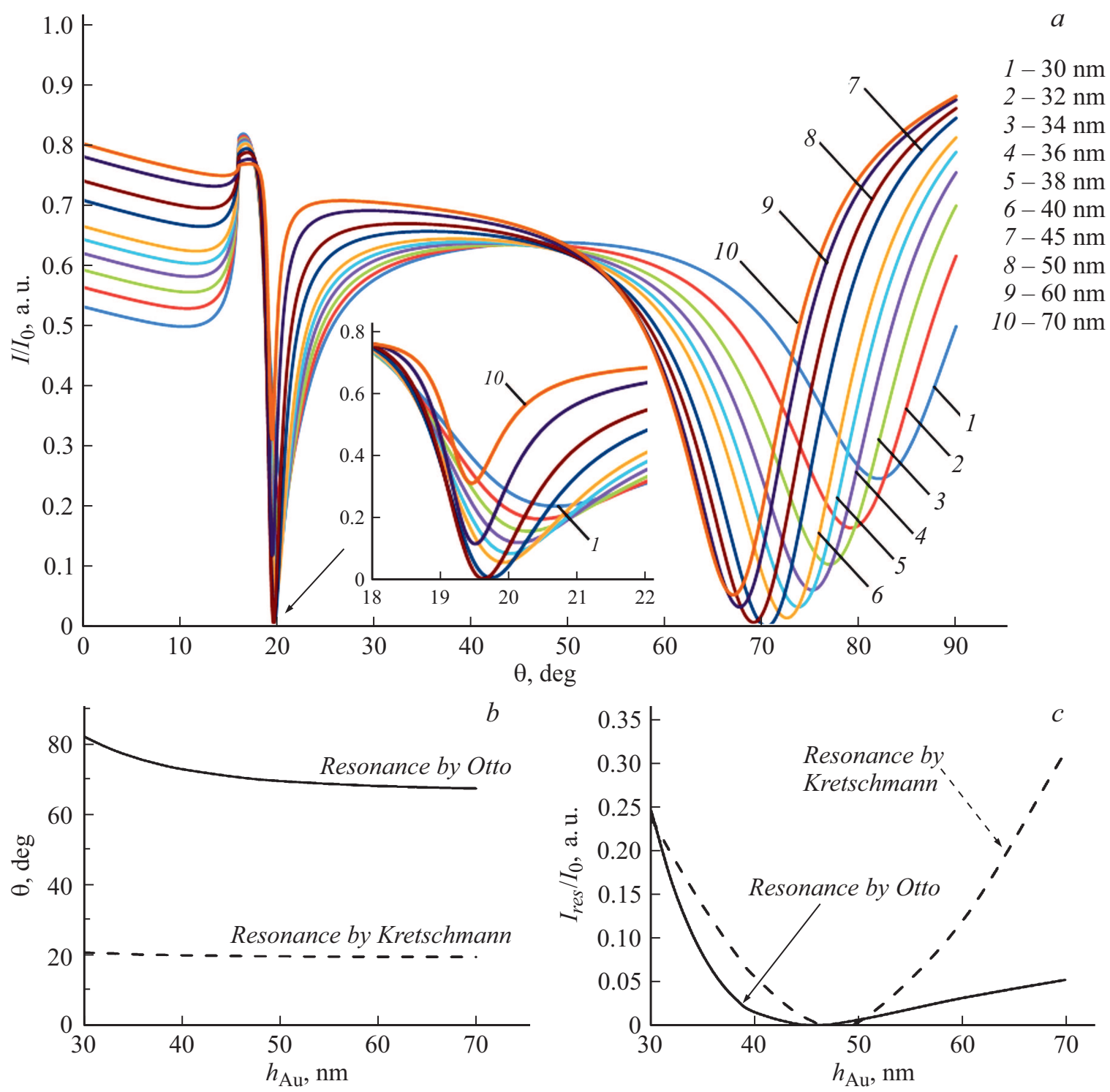

Рис. 3. Влияние толщины плазмонного слоя $h_{\mathrm{Au}}$ на резонансные свойства плазмонной структуры $\mathrm{GGG}$ prism $/ \mathrm{SiO}_{2}(200) / \mathrm{Au}$ : $a$ - угловые зависимости интенсивности отраженного луча $I$ нормированной на интенсивность падающего луча $I_{0}\left(h_{\text {Au }}\right.$ указана в легенде $) ; b-$ зависимость резонансных углов $\theta_{\mathrm{Kr}}\left(h_{\mathrm{Au}}\right)$ и $\theta_{\mathrm{Oto}}\left(h_{\mathrm{Au}}\right) ; c-$ нормированные интенсивности резонансных сигналов $I_{\text {res }}\left(h_{\mathrm{Au}}\right) / I_{0}$.

больших углах падения - в соответствии с конфигурацией Отто.

В данном случае толщина буферного слоя варьируется в диапазоне $100-300 \mathrm{~nm}$, толщина плазмонного слоя $\mathrm{Au}$ является фиксированной $h_{\mathrm{Au}}=50 \mathrm{~nm}$, диэлектрическая проницаемость внешней среды $\varepsilon_{\mathrm{env}}=1$.

На рис. $2, b$ представлена зависимость резонансного угла, а на рис. 2,c - зависимость нормированного резонансного сигнала от толщины буферного слоя $\mathrm{SiO}_{2}$ (для обоих резонансов). Как видно из представленных рисунков, толщина буферного слоя слабо влияет на свойства резонанса по Кретчману, однако существенно влияет на свойства резонанса по Отто, при этом меняется как положение резонансного пика, так и добротность резонанса. Так для малых толщин буфера
$(100-140 \mathrm{~nm})$ резонанс по Отто имеет низкую добротность, характеризуется слабой степенью возбуждения ППП и наблюдается при больших углах падения. По мере увеличения толщины слоя добротность резонанса увеличивается, а положение резонансного пика смещается в область меньших углов. Наиболее „качественный“ по добротности и эффективности возбуждения ППП резонанс наблюдается при толщине буферного слоя $200-220 \mathrm{~nm}$. Дальнейшее увеличение толщины буфера приводит к уменьшению эффективности возбуждения ППП по схеме Отто, при этом положение резонанса на резонансной кривой практически не меняется.

Анализ влияния толщины плазмонного слоя $\mathrm{Au}$ на резонансные свойства плазмонной структуры показан на рис. 3. В данном случае толщина плазмонного слоя $\mathrm{Au}$ 

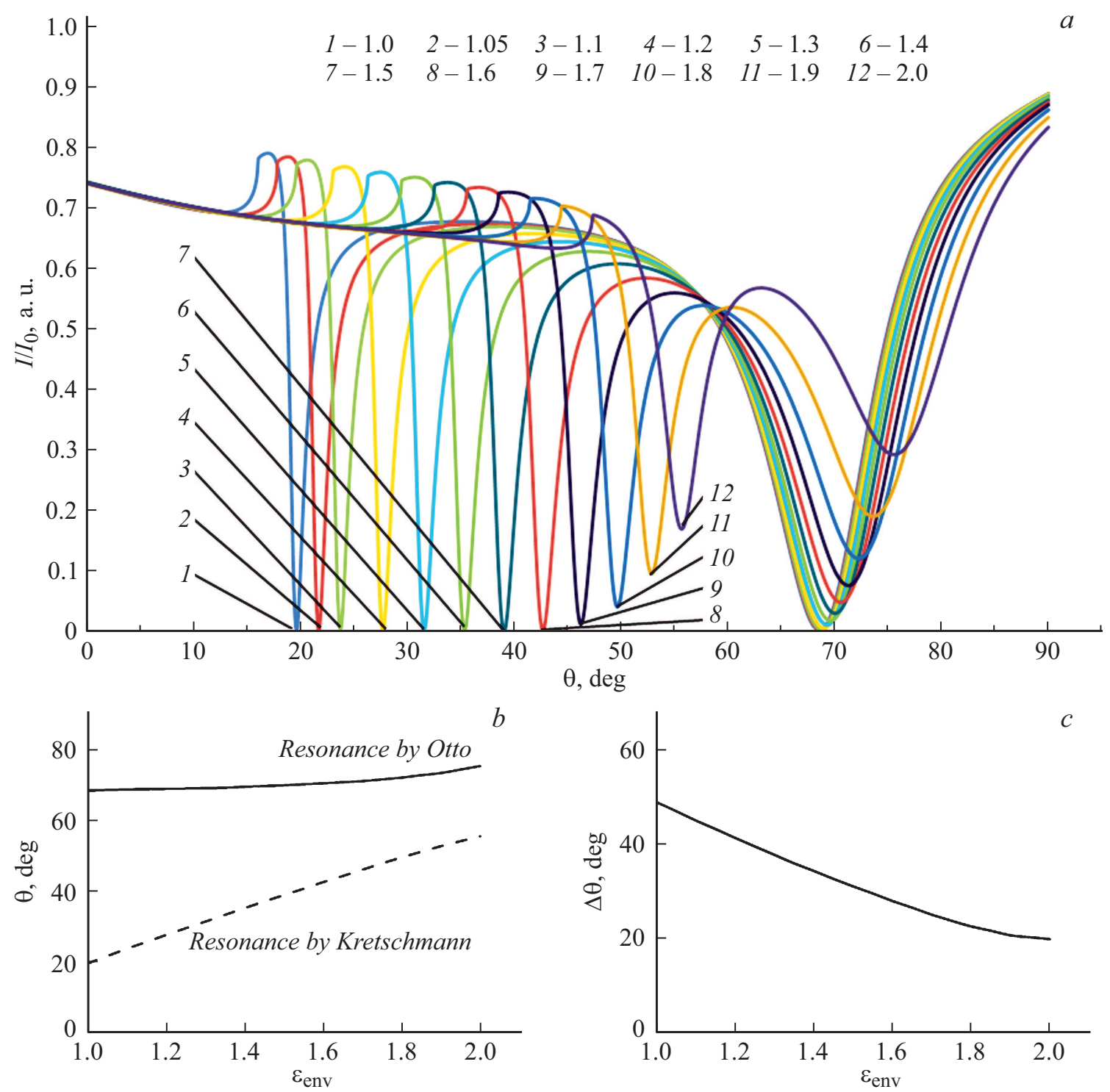

Рис. 4. Влияние диэлектрической проницаемости внешней среды $\varepsilon_{\text {env }}$ на резонансные свойства плазмонной структуры $\mathrm{GGG}_{\text {prism }} / \mathrm{SiO}_{2}(220) / \mathrm{Au}(50): a-$ угловые зависимости интенсивности отраженного луча $I$, нормированной на интенсивность падающего луча $I_{0}\left(\varepsilon_{\text {env }}\right.$ указана в легенде $) ; b-$ зависимость резонансных углов $\theta_{\mathrm{Kr}}\left(\varepsilon_{\text {env }}\right)$ и $\theta_{\text {Otto }}\left(\varepsilon_{\text {env }}\right) ; c-$ относительный сдвиг резонансов $\Delta \theta\left(\varepsilon_{\text {env }}\right)=\theta_{\text {Otto }}\left(\varepsilon_{\text {env }}\right)-\theta_{\mathrm{Kr}}\left(\varepsilon_{\text {env }}\right)$.

варьируется в диапазоне от 30 до $70 \mathrm{~nm}$, а толщина буферного слоя является фиксированной и составляет $200 \mathrm{~nm}$.

Из анализа резонансных кривых на рис. 3, $a$ следует, что толщина плазмонного слоя существенно влияет на параметры обоих резонансов. При этом положение резонанса по Кретчману меняется слабо (около $1.5 \mathrm{deg}$ ), а положение резонанса по Отто существенно смещается на резонансной кривой в сторону меньших углов при увеличении толщины плазмонного слоя (рис. $3, b$ ). Изменение добротности и эффективности возбуждения ППП (глубины резонансного пика) имеет схожее поведение для обоих резонансов (рис. 3,c). Наибольшая эффективность возбуждения ППП для обоих резонансов наблюдается при толщине $h_{\mathrm{Au}}=45-50 \mathrm{~nm}$. При даль- нейшем увеличении толщины слоя Аu оба резонансных пика становятся более узкими, при этом пик резонанса по Кретчману резко уменьшается по высоте, а пик резонанса по Отто стремится к некоторому стационарному виду.

Таким образом, на основе результатов моделирования предлагается следующая оптимальная конфигурация двухрезонансной структуры, совмещающей конфигурации Отто и Кретчмана, для возбуждения различных мод ППП. На гипотенузную грань прямоугольной равнобедренной призмы, изготовленной из монокристалла GGG, напыляется буферный слой кварца $\left(\mathrm{SiO}_{2}\right)$ толщиной 200-220 nm. Поверх буферного слоя напыляется плазмонный слой золота (Au, не хуже 99.95\%) толщиной $45-50 \mathrm{~nm}$. 


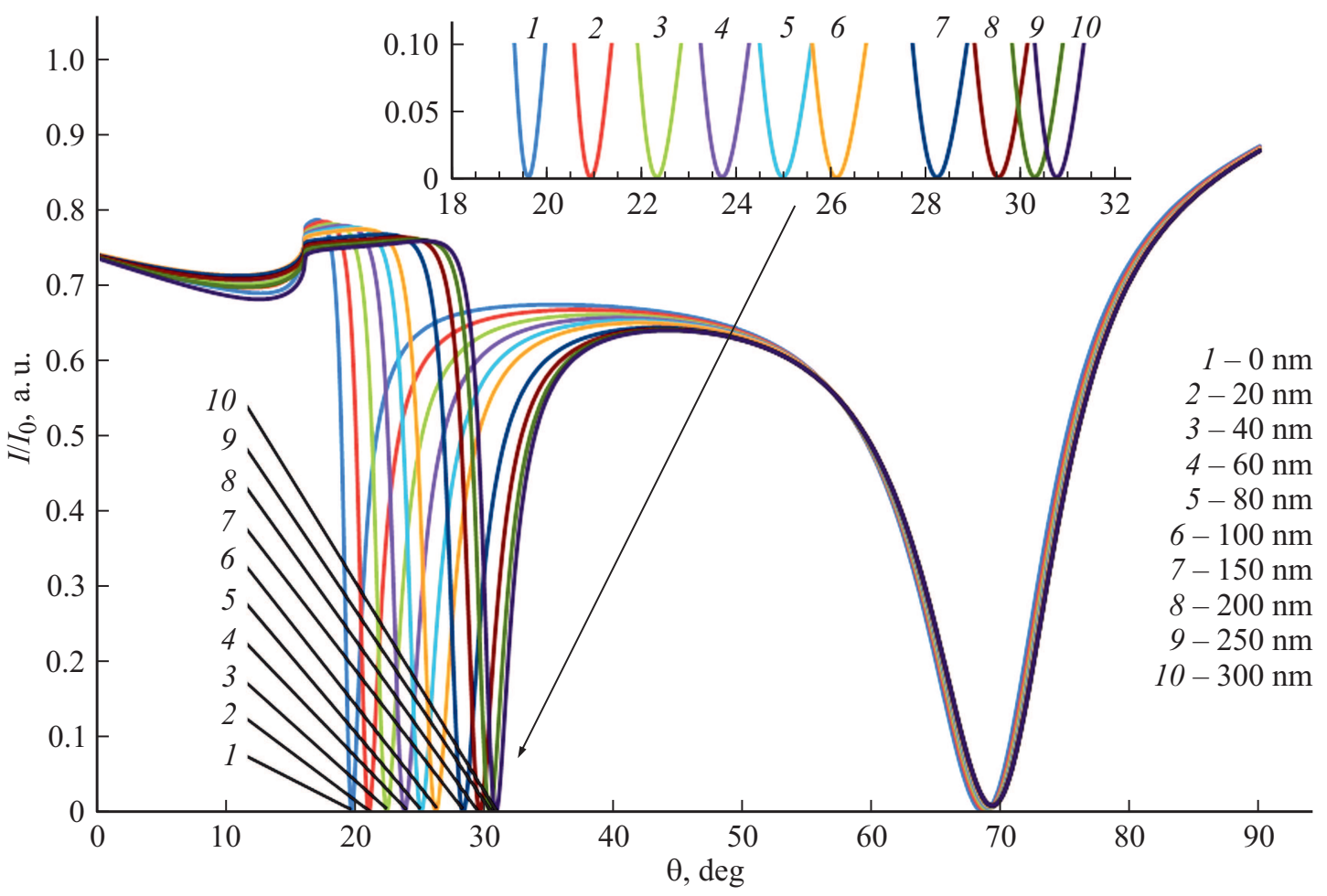

Рис. 5. Влияние толщины лигандного слоя $h_{\mathrm{Lg}}$ (указана в легенде) на резонансные свойства плазмонной структуры $\mathrm{GGG}_{\text {prism }} / \mathrm{SiO}_{2}(220) / \mathrm{Au}(50) / \mathrm{Lg}$ (угловые зависимости интенсивности отраженного луча I, нормированной на интенсивность падающего луча $\left.I_{0}\right)$.

\section{2. Анализ влияния параметров внешней среды на изменение резонансных условий возбуждения различных мод ППП в двухрезонансной структуре}

Рассмотрим модель двухрезонансной структуры $\mathrm{GGG}_{\text {prism }} / \mathrm{SiO}_{2}(220) / \mathrm{Au}(50)$ с толщиной буферного слоя $220 \mathrm{~nm}$ и толщиной плазмонного слоя $50 \mathrm{~nm}$. В качестве изменяемого параметра выступает действительная часть диэлектрической проницаемости внешней среды $\varepsilon_{\text {env }}$ (рис. 4). Подобная модель демонстрирует использование двухрезонансной плазмонной структуры в качестве чувствительного элемента плазмонного сенсора.

Как видно из резонансных кривых (рис. 4, $a$ ), изменение диэлектрической проницаемости внешней среды $\varepsilon_{\mathrm{env}}$ существенно влияет на изменение резонансных свойств двухрезонансной структуры. При малых значениях $\varepsilon_{\mathrm{env}}$ $\left(\varepsilon_{\text {env }} \ll \varepsilon_{\mathrm{SiO}_{2}}\right)$ резонансный пик по Кретчману испытывает сильное смещение на резонансной кривой. Так, изменение $\varepsilon_{\text {env }}$ на 0.05 (что соответствует изменению показателя преломления примерно на 0.025$)$ приводит к смещению резонансного пика по Кретчману на $2 \mathrm{deg}$, т.е. на ширину пика на полувысоте. В то же время пик резонанса по Отто весьма слабо изменяет свое положение (рис. $4, b$ ) вплоть до значения $\varepsilon_{\mathrm{env}}=1.6$. При дальнейшем увеличении $\varepsilon_{\text {env }}$ по мере его приближения к значению $\varepsilon_{\mathrm{SiO}_{2}}$ пик резонанса по Отто также начинает смещаться.
На рис. 4,c показана зависимость от $\varepsilon_{\text {env }}$ относительного положения (сдвига) резонансных пиков Отто и Кретчмана на рефлектометрической кривой $\Delta \theta\left(\varepsilon_{\text {env }}\right)=\theta_{\text {Otto }}\left(\varepsilon_{\text {env }}\right)-\theta_{\mathrm{Kr}}\left(\varepsilon_{\text {env }}\right)$. Видно, что при значениях $\varepsilon_{\mathrm{env}} \rightarrow \varepsilon_{\mathrm{SiO}_{2}}$ расстояние между резонансными пиками становится постоянным, т. е. оба пика испытывают одинаковое смещение. Таким образом, предложенная двухрезонансная структура способна эффективно работать в роли плазмонного сенсора по принципу сравнения положений двух резонансных пиков только при условии $\varepsilon_{\mathrm{env}}<\varepsilon_{\mathrm{SiO}_{2}}$ (в нашем случае $\left.1<\varepsilon_{\mathrm{env}}<1.6\right)$. Анализ показывает, что изменение $\varepsilon_{\text {env }}$ на 0.05 приводит к изменению расстояния между сенсорным (по Кретчману) и опорным (по Отто) резонансными пиками на $1.9 \mathrm{deg}$. Это соответствует чувствительности датчика $80.1 \mathrm{deg} / \mathrm{RIU}$ (refractive index unit).

\section{3. Анализ влияния параметров внешнего слоя на изменение резонансных условий возбуждения различных мод ППП в двухрезонансной структуре (аналог плазмонного биосенсора)}

В данной части работы моделировалось влияние толщины внешнего диэлектрического слоя $h_{\mathrm{Lg}}$ (лиганда в плазмонном биосенсоре) на особенности резонансного возбуждения различных мод ППП в предложенной плазмонной структуре $\mathrm{GGG}_{\text {prism }} / \mathrm{SiO}_{2}(220) / \mathrm{Au}(50) / \mathrm{Lg}$ (тол- 

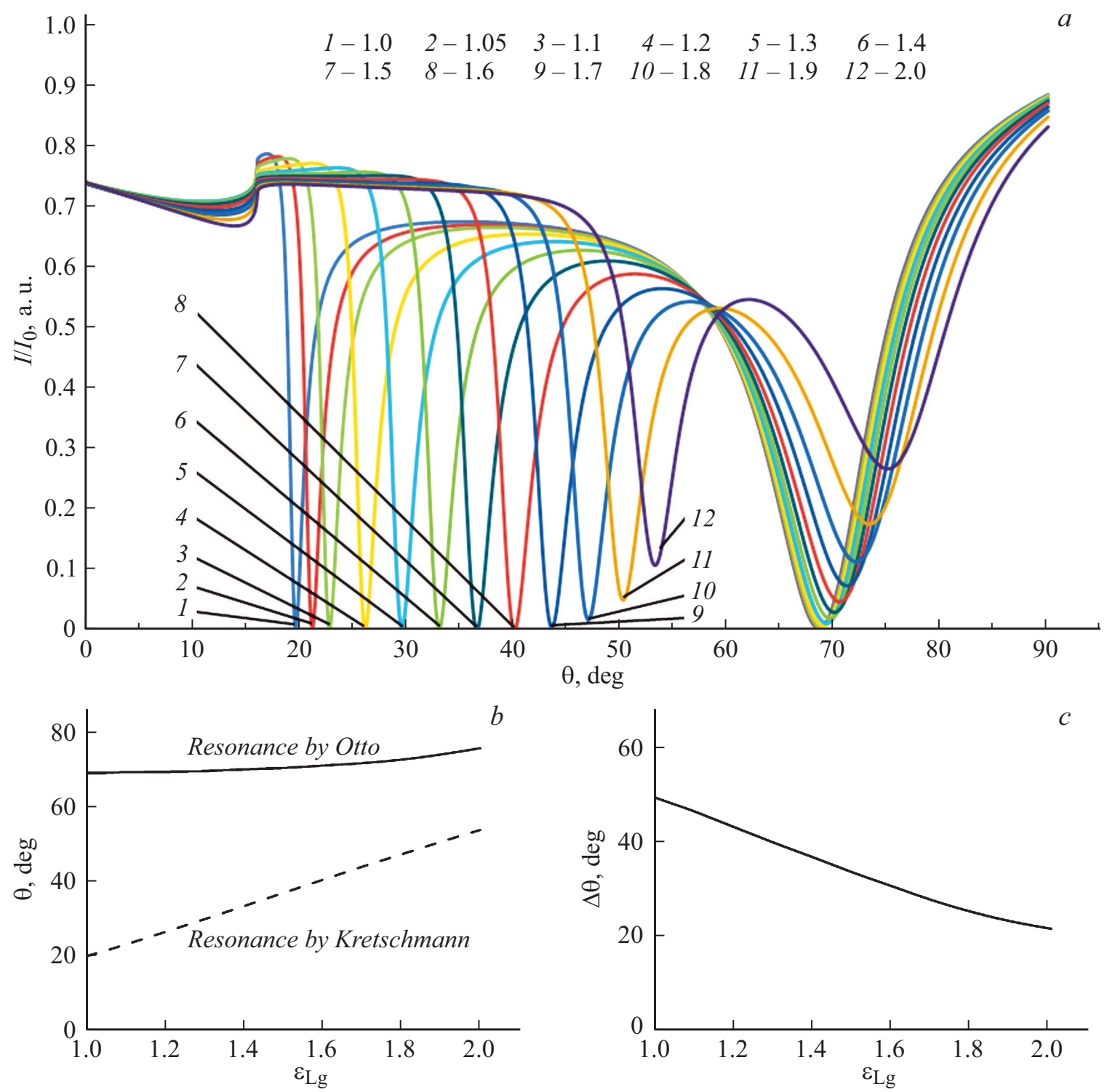

Рис. 6. Влияние диэлектрической проницаемости внешнего лигандного слоя $\varepsilon_{\mathrm{Lg}}$ на резонансные свойства плазмонной структуры $\mathrm{GGG}_{\text {prism }} / \mathrm{SiO}_{2}(220) / \mathrm{Au}(50) / \mathrm{Lg}(200): a$ - угловые зависимости интенсивности отраженного луча $I$, нормированной на интенсивность падающего луча $I_{0}\left(\varepsilon_{\mathrm{Lg}}\right.$ указана в легенде); $b-$ зависимость резонансных углов $\theta_{\mathrm{Kr}}\left(\varepsilon_{\mathrm{Lg}}\right)$ и $\theta_{\mathrm{Otto}}\left(\varepsilon_{\mathrm{Lg}}\right) ; c-$ относительный сдвиг резонансов $\Delta \theta\left(\varepsilon_{\mathrm{Lg}}\right)=\theta_{\mathrm{Otto}}\left(\varepsilon_{\mathrm{Lg}}\right)-\theta_{\mathrm{Kr}}\left(\varepsilon_{\mathrm{Lg}}\right)$.

щина буферного слоя $220 \mathrm{~nm}$, толщина плазмонного слоя $50 \mathrm{~nm})$. Диэлектрическая проницаемость лиганда $\varepsilon_{\mathrm{Lg}}=1.3$, а внешней среды $\varepsilon_{\mathrm{env}}=1$. Толщина лигандного слоя $h_{\mathrm{Lg}}$ изменялась в диапазоне от 0 (непосредственно сама структура) до $300 \mathrm{~nm}$. Результаты модельного анализа зависимости интенсивности отраженного луча от угла падения при разных значениях толщины лигандного слоя $h_{\mathrm{Lg}}$ показаны на рис. 5 .

Как видно из рисунка, увеличение толщины лигандного слоя $h_{\mathrm{Lg}}$ приводит к смещению пика резонанса по Кретчману, при этом пик резонанса по Отто практически не меняет своего положения на рефлектометрической кривой. Следует отметить, что изменение толщины лигандного слоя свыше $200 \mathrm{~nm}$ слабо изменяет положение резонансного пика по Кретчману, поэтому использование слоев с толщинами свыше $200 \mathrm{~nm}$ является избыточным.

Результаты модельного анализа влияния диэлектрической проницаемости внешнего лигандного слоя $\varepsilon_{\mathrm{Lg}}$ на резонансные свойства двухрезонансной структуры $\mathrm{GGG}_{\text {prism }} / \mathrm{SiO}_{2}(220) / \mathrm{Au}(50) / \mathrm{Lg}(200)$ представлены на рис. 6. В данной модели толщина внешнего слоя $h_{\mathrm{Lg}}$ составляла $200 \mathrm{~nm}$, а $\varepsilon_{\mathrm{Lg}}$ изменялась в диапазоне от 1 до 2.

На рис. 6, а представлены резонансные кривые при различном значении $h_{\mathrm{Lg}}$ (указана в легенде). На рис. $6, b$ показана зависимость резонансного угла для обоих резонансов от $\varepsilon_{\mathrm{Lg}}$, а на рис. $6, c$ - зависи- 

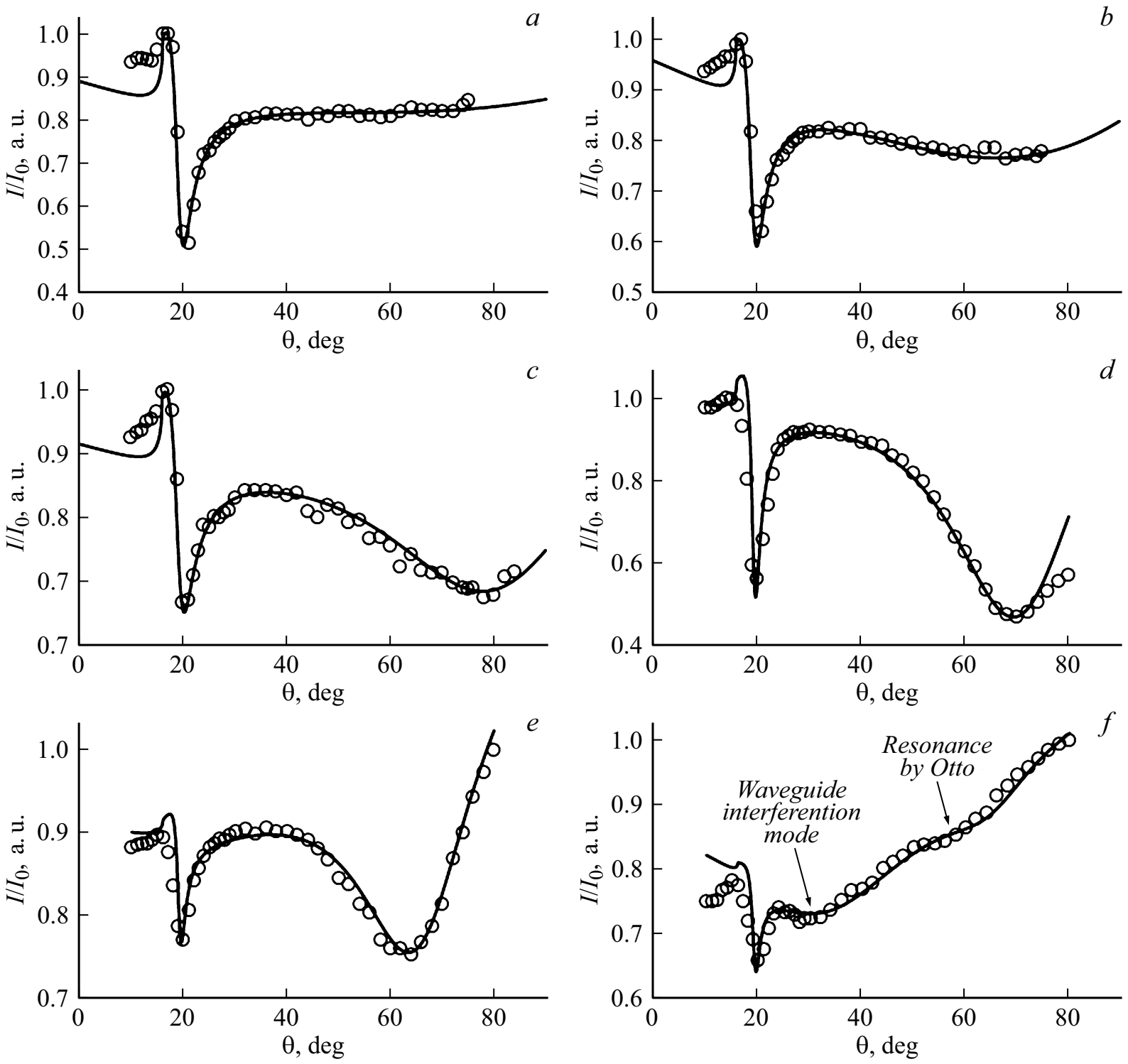

Рис. 7. Резонансные свойства синтезированной двухрезонансной плазмонной структуры $\mathrm{GGG}_{\text {prism }} / \mathrm{SiO}_{2}(\operatorname{grad} h) / \mathrm{Au}(50)$ на различных участках градиента толщины буферного слоя $h_{\mathrm{SiO}_{2}}: a-20, b-70, c-125, d-160, e-220 \mathrm{~nm}$; $f-350 \mathrm{~nm}($ угловые зависимости интенсивности отраженного луча $I$, нормированной на интенсивность падающего луча $I_{0}$ : круги - экспериментальные точки, сплошная линия - моделирование в пакете WinSpall).

мость относительного положения (сдвига) резонансных пиков $\Delta \theta\left(\varepsilon_{\mathrm{Lg}}\right)=\theta_{\mathrm{Otto}}\left(\varepsilon_{\mathrm{Lg}}\right)-\theta_{\mathrm{Kr}}\left(\varepsilon_{\mathrm{Lg}}\right)$. Видно, что характер изменения условий резонансного возбуждения различных мод ППП в плазмонной структуре $\mathrm{GGG}_{\text {prism }} / \mathrm{SiO}_{2}(220) / \mathrm{Au}(50) / \mathrm{Lg}(200)$ при изменении $\varepsilon_{\mathrm{Lg}}$ практически аналогичен изменению условий возбуждения ППП в структуре $\mathrm{GGG}_{\text {prism }} / \mathrm{SiO}_{2}(220) / \mathrm{Au}(50)$ при изменении диэлектрической проницаемости внешней среды $\varepsilon_{\mathrm{env}}$ (рис. 4). Отличие наблюдается лишь в изменении угла ПВО. Таким образом, видно, что предложенная двухрезонансная плазмонная структура способна эффективно детектировать изменение диэлектрической проницаемости как внешней среды (плазмонный сенсор), так и тонких диэлектрических слоев (лигандов), нанесенных на поверхность плазмонного покрытия (плазмонный биосенсор).

\section{4. Экспериментальное изготовление и исследование двухрезонансной структуры для возбуждения различных мод ППП}

Для проверки условий резонансного возбуждения различных плазмон-поляритонных мод была изготовлена двухрезонансная структура $\mathrm{GGG}_{\text {prism }} / \mathrm{SiO}_{2}(\operatorname{grad} h) / \mathrm{Au}(50)$ с расчетной толщиной слоя золота $50 \mathrm{~nm}$ и градиентом толщины буферного слоя $h_{\mathrm{SiO}_{2}}$. Толщина буферного слоя $h_{\mathrm{SiO}_{2}}$ изменялась в пределах от 20 до $350 \mathrm{~nm}$, исследо- 
вание осуществлялось в различных точках градиента. На рис. 7 показаны рефлектометрические резонансные кривые, измеренные на изготовленной структуре $\mathrm{GGG}_{\text {prism }} / \mathrm{SiO}_{2}(\operatorname{grad} h) / \mathrm{Au}(50)$ при различной толщине буферного слоя $h_{\mathrm{SiO}_{2}}$.

Видно, что при малой толщине буферного слоя $h_{\mathrm{SiO}_{2}}=20 \mathrm{~nm}$ (рис. 7, $a$ ) на рефлектометрической кривой наблюдается только один пик резонанса по Кретчману (на рисунке пустые кружки соответствуют экспериментальным результатам, сплошная линия - моделирование в программе WinSpall). Резонансный угол при этом составляет $19.5 \mathrm{deg}$ и незначительно меняется в дальнейшем при изменении $h_{\mathrm{SiO}_{2}}$, как и было показано ранее при моделировании (рис. 2).

Увеличение толщины буферного слоя до $70 \mathrm{~nm}$ (рис. 7,b) приводит к возникновению локального минимума, соответствующего поглощению энергии на резонансное возбуждение плазмонных волн по механизму Отто, однако резонанс имеет крайне низкую добротность и малую эффективность возбуждения ППП. Увеличение толщины буферного слоя $h_{\mathrm{SiO}_{2}}$ до $125 \mathrm{~nm}$ (рис. 7, c) приводит к увеличению глубины резонансного пика (возрастает эффективность возбуждения ППП) и добротности резонанса по Отто. В данном случае имеется возможность оценить резонансный угол пика по Отто, который составляет около $79 \mathrm{deg}$. При толщине буферного слоя $h_{\mathrm{SiO}_{2}}=160 \mathrm{~nm}$ (рис. 7,d) четкий пик плазмон-поляритонного резонанса по Отто наблюдается при угле падения возбуждающего пучка $69.5 \mathrm{deg}$.

Дальнейшее увеличение толщины буферного слоя до $220 \mathrm{~nm}$ (рис. 7,e) приводит к возрастанию добротности резонанса, при этом резонанс по Отто наблюдается при угле падения луча $64 \mathrm{deg}$. При этом несколько снижается эффективность возбуждения ППП по механизму Кретчмана, что связано, очевидно, с частичным рассеянием излучения внутри буферного слоя и поглощением части энергии на возбуждение плазмонных колебаний по механизму Отто.

Как и было показано при моделировании, дальнейшее увеличение толщины $h_{\mathrm{SiO}_{2}}$ приводит к уменьшению высоты резонансного пика по Отто. Так, при $h_{\mathrm{SiO}_{2}}=350 \mathrm{~nm}$ (рис. 7,f) резонансный пик по Отто становится слаборазличим, при этом буферный слой $\mathrm{SiO}_{2}$ приобретает свойства планарного оптического волновода и на рефлектометрической зависимости вблизи угла падения светового луча $30 \mathrm{deg}$ наблюдается локальный минимум, связанный с появлением интерференционных волноводных мод [14].

Результаты, представленные на рис. 7, наглядно показывают возможность практической реализации предложенной двухрезонансной плазмонной структуры, для которой возможно возбуждение различных мод ППП за счет совмещения конфигураций Отто и Кретчмана. Результаты демонстрируют хорошее совпадение экспериментальных данных с модельными расчетами.

\section{Заключение}

Таким образом, в работе теоретически и экспериментально была продемонстрирована возможность создания двухрезонансной структуры для возбуждения различных мод ППП волн посредством совмещения конфигураций Кретчмана и Отто на одном устройстве. Модельная оптимизация параметров показала, что для предложенной структуры $\mathrm{GGG}_{\text {prism }} / \mathrm{SiO}_{2} / \mathrm{Au}$ оптимальная толщина буферного слоя $\mathrm{SiO}_{2}$ составляет 200-220 nm, а толщина плазмонного слоя Аu составляет 45-50 nm.

Анализ влияния диэлектрической проницаемости внешней среды $\varepsilon_{\text {env }}$ на особенности резонансного возбуждения различных мод ППП (аналог плазмонного сенсора) показал, что изменение вещественной части диэлектрической проницаемости окружающей среды $\varepsilon_{\mathrm{env}}$ на 0.05 приводит к смещению резонансного пика по Кретчману на $2 \mathrm{deg}$. Пик резонанса по Отто при этом практически не смещается и может быть использован в качестве опорного в схеме сравнения положения двух пиков. Для такой схемы сравнения чувствительность плазмонного датчика составляет $80.1 \mathrm{deg} / \mathrm{RIU}$. Результаты моделирования работы предложенной структуры $\mathrm{GGG}_{\text {prism }} / \mathrm{SiO}_{2} / \mathrm{Au} / \mathrm{Lg}$ в качестве плазмонного биосенсора с внешним чувствительным слоем (лигандом) показали, что использование лигандного слоя свыше $200 \mathrm{~nm}$ является избыточным, при этом изменение вещественной части диэлектрической проницаемости слоя $\varepsilon_{\mathrm{Lg}}$ приводит к изменению положения резонансных пиков аналогично по сравнению с изменением $\varepsilon_{\text {env }}$.

Результаты экспериментального изготовления и исследования предложенной двухрезонансной структуры $\mathrm{GGG}_{\text {prism }} / \mathrm{SiO}_{2}(\operatorname{grad} h) / \mathrm{Au}(50)$ для возбуждения различных мод ППП полностью подтвердили результаты модельных расчетов. При этом было показано, что для сверхмалых толщин $h_{\mathrm{SiO}_{2}}$ диэлектрического буферного слоя резонанс по Отто не наблюдается (резонанс по Кретчману наблюдается при всех значениях $h_{\mathrm{SiO}_{2}}$ ), далее по мере увеличения толщины буферного слоя резонанс по Отто обнаруживается и достигает максимальной добротности и эффективности возбуждения ППП при толщине $h_{\mathrm{SiO}_{2}}=220 \mathrm{~nm}$. Дальнейшее увеличение $h_{\mathrm{SiO}_{2}}$ приводит к деградации резонансного пика по Отто и при толщинах буферного слоя $350 \mathrm{~nm}$ и более резонанс по Отто исчезает, и начинают наблюдаться интерференционные волноводные моды.

\section{Финансирование работы}

Работа выполнена при финансовой поддержке гранта Госсовета Республики Крым, пост. № п66-2/20 от 04.02.2020.

\section{Конфликт интересов}

Авторы заявляют, что у них нет конфликта интересов. 


\section{Список литературы}

[1] С.А. Майер. Плазмоника: теория и приложения (НИЦ „Регулярная и хаотическая динамика“, М.-Ижевск, 2011)

[2] J.R. Mejia-Salazar, S.A. Camacho, C.J.L. Constantino, O.N. Oliveira. An. Acad. Bras. Cienc., 90, 779 (2018). DOI: $10.1590 / 0001-3765201820170571$

[3] B.A. Prabowo, A. Purwidyantri, K.-Ch. Liu. Biosensors, 8, 80 (2018). DOI: 10.3390/bios 8030080

[4] B. Sepulveda, A. Calle, L. M. Lechuga, G. Armelles. Opt. Lett., 31 (8), 1085 (2006). DOI: 10.1364/OL.31.001085

[5] Saiful I., Jakeya S., A.A. Rifat, R. Ahmed, A. Dinovitser, B.W.-H. Ng, H. Ebendorff-Heidepriem, D. Abbott. Opt. Express, 26 (23), 30347 (2018). DOI: 10.1364/OE.26.030347

[6] N.-H. Kim, M. Choi, T.W. Kim, W. Choi, S.Y. Park, K.M. Byun. Sensors, 19, 1894 (2019).

DOI: $10.3390 / \mathrm{s} 19081894$

[7] A. Otto. Zeitschrift für Physik A, 216 (4), 398 (1968).

[8] E. Kretschmann, H. Raether, Notizen. Zeitschrift für Naturforschung A, 23, 2135 (1968).

[9] K.M. McPeak, S.V. Jayanti, S.J.P. Kress, S. Meyer, S. Iotti, A. Rossinelli, D.J. Norris. ACS Photonic, 2 (3), 326 (2015). DOI: $10.1021 / \mathrm{ph} 5004237$

[10] С.В. Томилин, В.Н. Бержанский, А.Н. Шапошников, Г.Д. Басиладзе. Плазмон-поляритонный двухрезонансный датчик [Пат. № 169687 Россия, МПК G01N 21/63]. Бюл. № 6 (2017).

[11] L. Lixia, L. Yuzhang, G. Jianye, C. Wenli, Z. Xinpu, J.-F. Masson, P. Wei. Opt. Express, 25 (22), 26950 (2017). DOI: $10.1364 / \mathrm{OE} .25 .026950$

[12] Д.А. Мамичев, И. А. Кузнецов, Н.Е. Маслова, М.Л. Занавескин. Молекулярная медицина, 6, 19 (2012).

[13] С.В. Томилин, В.Н. Бержанский, А.С. Яновский, О.А. Томилина. Поверхность. Рентгеновские, синхротронные и нейтронные исследования, 8, 96 (2016).

DOI: $10.7868 / \mathrm{S} 0207352816080187$

[S.V. Tomilin, V.N. Berzhansky, A.S. Yanovsky, O.A. Tomilina. J. Surf. Investigation: X-ray, Synchrotron and Neutron Techniq., 10 (4), 868 (2016).

DOI: $10.1134 /$ S1027451016040376]

[14] L.B. Soldano, E.C.M. Pennings. J. Lightwave Technol., 13 (4), 615 (1995). 